\title{
The mean compression stress of reinforced honeycomb under quasi-static compression
}

\author{
Song Yang ${ }^{1, a}$, Zhao Hui ${ }^{1, b}$,Luo Changjie ${ }^{2, \mathrm{c}}$,Liu Rongqiang ${ }^{3, \mathrm{~d}}$ \\ ${ }^{1}$ Northeast Forestry University, China \\ ${ }^{2}$ Shenzhen Cansinga Technology CO.,Ltd,China \\ ${ }^{3}$ Harbin Institute of Technology, China \\ aysong0727@126.com, bzhaohv@163.com, ${ }^{\text {c }}$ cj.luo@cansinga.com, ${ }^{\mathrm{d}}$ liurq6507@126.com
}

\begin{abstract}
Keywords: reinforced honeycomb, axial quasi-static compression, mean stress, compression simulation

Abstract. To improve the strength of hexagonal honeycomb, the structure of reinforced honeycomb is proposed in this paper. The strength of this structure could be adjusted by changing the superposition mode of core material. The theoretical model of reinforced honeycomb is built based on the theory of simplified basic folding element to research about the relationship between superposition mode and the crushing strength. The FEM software MSC.patran is used to simulate the process of quasi-static compression with different modes, in addition, the quasi-static compression experiments are executed on the universal testing machine. The results show that the compression strength is varied with different superposition modes. The results of simulation, theory and experiment are in good agreement.
\end{abstract}

\section{Introduction}

Honeycomb is a kind of porous cellular structure, which is widely used in the field of aeronautics and aerospace, rail traffic passive safety as a kind of ideal crash buffer to absorb the collision energy and thus reduce injury, by the advantages of its light mass, stable compression load, large compression rate, et al. However common hexagon honeycomb can't satisfy the strength requirement in some collision condition. In the process of honeycomb forming by formation method, a flat aluminum foil is added between two corrugated sheets to form reinforced honeycomb which improves the compression strength, this kind of structure is called reinforced honeycomb. $\mathrm{He}^{[1]}$ investigated the effects of corrugated sheet thickness and flat foil thickness on the energy absorption mass ratio in reinforced honeycomb, meanwhile he found that impact velocity has great influence on stress-strain curve. Yin ${ }^{[2]}$ built the mean stress theoretical model of hexagonal honeycomb based on the simplified basic folding element theory.

Many researchers have studied the quasi static collapse stress characteristics of the honeycomb structures. Wierzbicki ${ }^{[3]}$ put forward the super folding element theory for thin-wall square tube and studied the capacity of energy-absorbing during the compression deformation of folding element. He also derived the mean stress formula under static compression. $\mathrm{Li}^{[4]}$ selected the "Y" type cell to compare the theoretical models of mean stress based on the criteria of Tresca, Mises and Wierzbicki. Sun $^{[5]}$ established the model of honeycomb under out-plane static compression to study the peak stress with different wall thickness using ANSYS/LS-DYNA. Bai ${ }^{[6]}$ applied ABAQUS to study the influence of honeycomb materials and cell length on the axial compression. $\mathrm{Ca}^{\left[{ }^{[7]}\right.}$ described the relationship between wall thickness and energy absorption mass ratio.

Different configurations of reinforced honeycomb is discussed in this paper, whose strength is adjusted by changing the number of layers of corrugated sheet between two flat sheets which is used to enhanced strength. The theoretical models of mean compression stress for seven configurations of reinforced honeycomb are established and their quasi-static compression processes are simulated. The experiments are conducted under static compression to verify the analysis of theory and simulation. 


\section{Reinforced honeycomb structure}

In this paper, the honeycomb is formed by rolling formation method ${ }^{[8]}$. The aluminum foil sheet is pressed into semi hexagonal corrugated structure at first, then the corrugated sheets are bonded by layer upon layer with special adhesive. The reinforced honeycomb is made by adding a flat aluminum foil which can enhance the honeycomb strength between two semi hexagonal corrugated sheets. The reinforced honeycomb is named according to the follow definition: reinforced honeycomb with $\mathrm{n}$ layers of corrugated sheets between two flat sheets is called " $n+1$ " type. HEXCEL Corporation put forward " $1+1$ " type reinforced honeycomb, whose structure diagram is shown in Fig. 1, where W is superimposition direction, $\mathrm{T}$ is axial direction. " $2+1 ", " 3+1 ", " 4+1$ " type are shown in Fig. 2, Fig. 3, and Fig. 4.

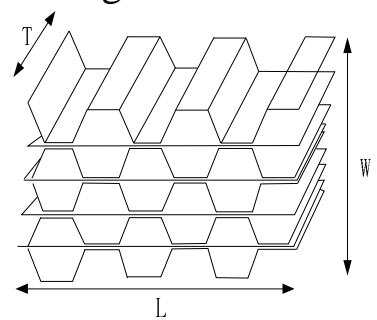

Fig. 1 Principal schematic of reinforced hexagon honeycomb

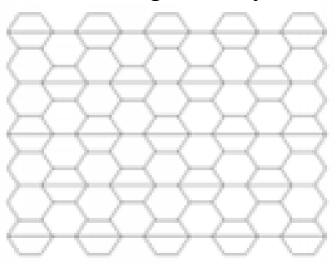

Fig. 3 " $3+1$ ” type structure

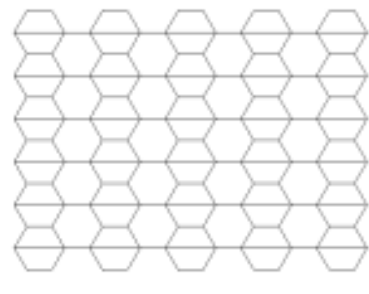

Fig. 2 " $2+1$ ” type structure

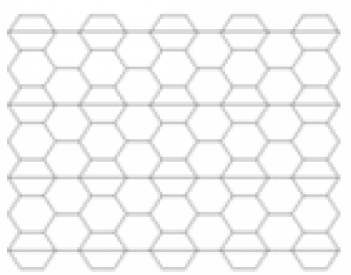

Fig. 4 "4+1" type structure

\section{Theoretical model of axial mean stress of honeycomb}

The regular hexagonal honeycomb

The axial section of hexagon honeycomb is described in Fig. 5a). The minimum period "Y" type cell is shown in Fig. 5b). A basic folding element whose wave length is $2 h$ in the compression process is shown in Fig. 5c).

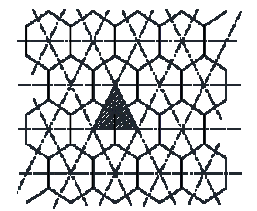

a) Regular honeycomb

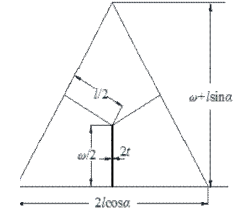

b) Minimum element

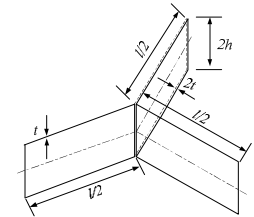

c) Basic folding element

Fig. 5 Axial structure of honeycomb

The absorbed energy of the above basic folding element in the compression process can be expressed as the sum of two extension elements energy absorption and three horizontal hinge elements energy absorption. Details about the basic folding element is shown in Fig. 6a), with wavelength $2 h$, horizontal hinge length $l / 2$ and wall thickness $t$.

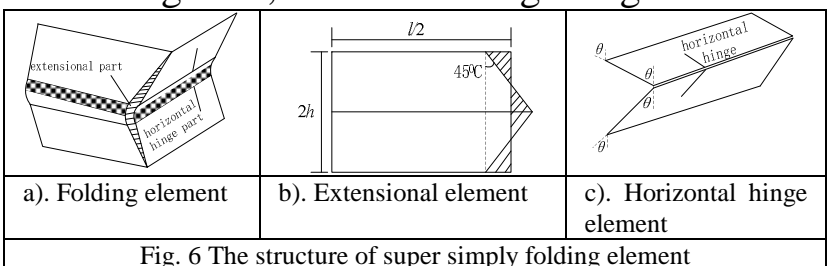

The principle of extension energy absorption is shown in Fig. 6b). The energy can be obtained by Eq. (1): 
$U_{m 1}=\int_{S} t \sigma_{0} \mathrm{dS}=\frac{1}{2} t \sigma_{0} h^{2}$

The horizontal hinge energy absorption is shown in Figure 6c). The hinge energy can be calculated by Eq. (2):

$$
U_{b 1}=\sum_{i=1}^{3} c M_{0} \theta_{i}=M_{0} \pi l=\frac{\sigma_{0} t^{2} \pi l}{4} .
$$

where $U_{m 1}$ and $U_{b 1}$ are the extension energy and horizontal hinge energy of above folding element respectively, and $\sigma_{0}$ is flow stress which is determined from Eq. (3):

$$
\boldsymbol{\sigma}_{0}=\sqrt{\frac{\boldsymbol{\sigma}_{y} \boldsymbol{\sigma}_{u}}{1+n}} .
$$

where $\sigma_{y}$ and $\sigma_{u}$ denote the yield strength and the ultimate strength of the material respectively, $\mathrm{n}$ is the exponent of the power law, $M_{0}$ is plastic bending moment of thin-wall honeycomb which can be evaluated by Eq. (4)

$$
M_{0}=\frac{\sigma_{0} t^{2}}{4} \text {. }
$$

The theoretical model of mean stress of " $1+1$ " type reinforced honeycomb under quasi-static compression

The structure of " $1+1$ " type reinforced honeycomb in axial direction is shown in Fig. 7a). The minimum period structure is shown in Fig. 7b). The basic folding element consists of four plates of folding length $l / 2$ and one plate of folding length $l$ which is shown in Fig. 7c).

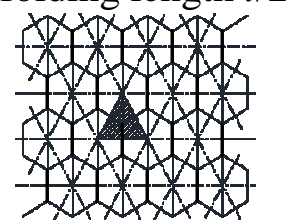

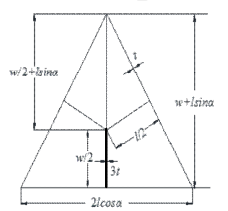

b) Minimum unit Fig. 7 the structure of super simply folding element

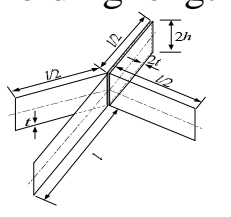

c) Basic folding unit

Setting axial compression rate of honeycomb as $\delta$, Eq. (5) can be written based on the conservation of energy for compression process of basic folding element:

$$
2 h \delta P_{m}=U_{m}+U_{b} .
$$

where $P_{m}$ is the mean crushing force, $U_{m}$ and $U_{b}$ are the extension energy and horizontal hinge energy in a complete folding element respectively. The extension energy of reinforced honeycomb can be calculated by Eq. (6)

$$
U_{m}=4 U_{m 1}=2 \sigma_{0} h^{2} t .
$$

The horizontal hinge energy can be calculated by Eq. (7) 


$$
\begin{aligned}
& U_{b}=2 U_{b 1}+U_{b 2}+U_{b 3} \\
& =2 \pi l \frac{\sigma_{0} t^{2}}{4}+\frac{\sigma_{0} t^{2}}{4}\left(\frac{\pi}{2} l+\pi l+\frac{\pi}{2} l\right)+\pi l \frac{\sigma_{0}(3 t)^{2}}{4} . \\
& =\frac{13}{4} \pi l_{0} t^{2}
\end{aligned}
$$

where $U_{b 2}$ and $U_{b 3}$ are the horizontal hinge energy element with hinge length $l$ and wall thickness $3 t$, respectively.

Substituting Eqs. (6) and (7) back into Eq. (5), Eq. (8) is given as follows by using the principle of minimum plastic deformation energy in the process of axial quasi-static compression, so Eq. (8) is required:

$$
\frac{\partial P_{m}}{\partial h}=0
$$

Then Eq. (9) is obtained:

$H=\sqrt{\frac{13 \pi l t}{8}}$.

Taking Eqs. (5),(6),(7) and (9), $P_{m}$ is evaluated as

$$
P_{m}=\frac{\sqrt{26 \pi t l}}{2 \delta} \sigma_{0}
$$

The area of basic folding element (the part of the shadow in Fig. 7a)) is calculated by $S=\frac{3 \sqrt{3}}{4} l^{2}$

With Eqs. (10) and (11), the mean stress of reinforced honeycomb is derived as

$$
\bar{\sigma}_{1+1}=\frac{2 \sqrt{26 \pi}}{3 \sqrt{3} \delta} \sigma_{0}\left(\frac{t}{l}\right)^{3 / 2} \text {. }
$$

where $\bar{\sigma}_{1+1}$ is the mean stress of " $1+1$ " type reinforced honeycomb.

By this method, the mean stress of regular hexagonal honeycomb can be obtained by

$$
\bar{\sigma}_{p t}=\frac{4 \sqrt{\pi}}{3 \delta} \sigma_{0}\left(\frac{t}{l}\right)^{3 / 2} \text {. }
$$

The theoretical model of mean stress of reinforced honeycomb with different configuration

The mean stress of " $1+1$ " type reinforced honeycomb can be expressed as Eq. (14):

$$
\bar{\sigma}_{1+1}=\bar{\sigma}_{p t}+\Delta \sigma
$$

where, $\Delta \sigma$ is the part caused by the flat sheets, which is as follow:

$$
\Delta \sigma=\bar{\sigma}_{1+1}-\bar{\sigma}_{p t} .
$$


For the " $2+1$ " type reinforced honeycomb, the number of the flat sheet is half of that of " $1+1$ " type reinforced honeycomb. Therefore, the mean stress of " $2+1$ " type is calculated by Eq. (16):

$$
\begin{aligned}
& \bar{\sigma}_{2+1}=\frac{1}{2} \Delta \sigma+\bar{\sigma}_{p t}=\frac{1}{2} \bar{\sigma}_{1+1}+\frac{1}{2} \bar{\sigma}_{p t} \\
& =\frac{1}{2}\left(\frac{2 \sqrt{26 \pi}+4 \sqrt{3 \pi}}{3 \sqrt{3} \delta}\right) \sigma_{0}\left(\frac{t}{l}\right)^{3 / 2}
\end{aligned}
$$

Similarly, the mean stress of " $3+1$ " type reinforced honeycomb can be expressed as follows:

$$
\begin{aligned}
& \bar{\sigma}_{3+1}=\frac{1}{3} \Delta \sigma+\bar{\sigma}_{p t}=\frac{1}{3} \bar{\sigma}_{1+1}+\frac{2}{3} \bar{\sigma}_{p t} \\
& =\frac{1}{3}\left(\frac{2 \sqrt{26 \pi}+8 \sqrt{3 \pi}}{3 \sqrt{3} \delta}\right) \sigma_{0}\left(\frac{t}{l}\right)^{3 / 2} .
\end{aligned}
$$

Based on the above method, the mean stress of " $n+1$ " type reinforced honeycomb is derived as Eq. (18):

$$
\overline{\boldsymbol{\sigma}}_{n+1}=\frac{1}{n} \overline{\boldsymbol{\sigma}}_{1+1}+\frac{n-1}{n} \overline{\boldsymbol{\sigma}}_{p t}
$$

\section{Quasi-static compression simulation by FEM}

Based on the FEM, the quasi-static compression process is simulated by MSC.Patran and LS-Dyna to validate the correctness of theoretical model.

Establishment of simulation model

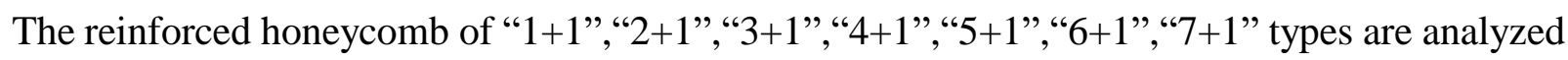
in the condition of same material, dimension of cell, thickness of aluminum foil sheet, and the raw material is aluminum allay h5052. The simulation model is built with the geometry dimension and mechanical parameters which are shown in Table 1 and Table 2, respectively.

\begin{tabular}{|c|c|c|c|c|}
\hline Tame & $\begin{array}{c}\text { cell length } \\
{[\mathrm{mm}]}\end{array}$ & $\begin{array}{c}\text { wall thickness } \\
{[\mathrm{mm}]}\end{array}$ & $\begin{array}{c}\text { height of model } \\
{[\mathrm{mm}]}\end{array}$ \\
\hline $\begin{array}{c}\text { numerical } \\
\text { value }\end{array}$ & 2.625 & 0.15 & 40 \\
\hline Table 2 The mechanical parameters of simulation model \\
\hline name & $\begin{array}{c}\text { material } \\
\text { density } \\
{\left[\mathrm{kg} / \mathrm{m}^{3}\right]}\end{array}$ & $\begin{array}{c}\text { elastic } \\
\text { modulus } \\
{[\mathrm{GPa}]}\end{array}$ & $\begin{array}{c}\text { poisson } \\
\text { ratio }\end{array}$ & $\begin{array}{c}\text { yield } \\
\text { strength } \\
{[\mathrm{MPa}]}\end{array}$ \\
\hline $\begin{array}{c}\text { numerical } \\
\text { value }\end{array}$ & 2680 & 70 & 0.33 & 255 \\
\hline
\end{tabular}

The model is shown in Fig. 8 which applies Balystchko Tsay shell element. Rigid 2 is used to simulate the hammer in constant speed. The friction coefficient is set to 0.17 between honeycomb and rigids, and the inner friction coefficient of honeycomb is set to 0.1 . The mesh is divided by quadrilateral elements in the form of 4 nodes.

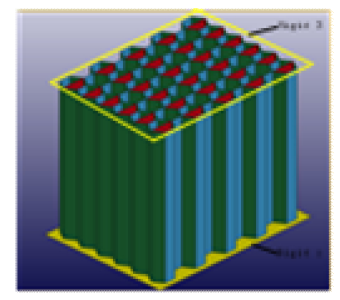

Fig. 8 The simulation model 


\section{Simulation results}

The processes of progressive compression of " $4+1$ " type honeycomb in different time are shown in Fig. 9. Noting that plastic bending deformation begins from bottom as the compression proceeds, and buckling fold is piling up until final collapse.
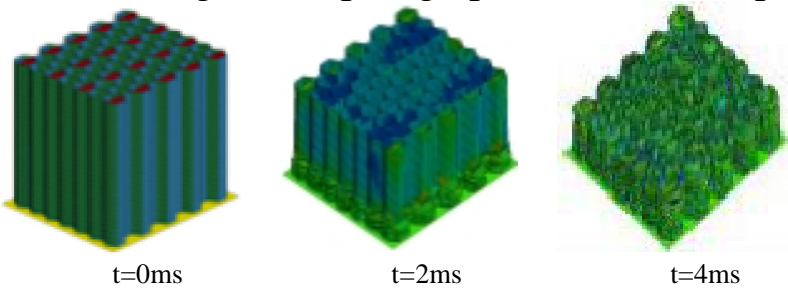

Fig. 9 Compressive deformation in different time

Fig. 10 is the stress-strain curve of reinforced honeycomb which indicates that the strength of honeycomb is changed with different number of layer plate. The curve suggests that " $1+1$ " type has the maximum strength, and " $7+1$ " type has the minimum one. With the interval of aluminum flat foil increasing, the strength decreases. There is no significant different in strength when the spacing of aluminum flat foil exceeds four layers.

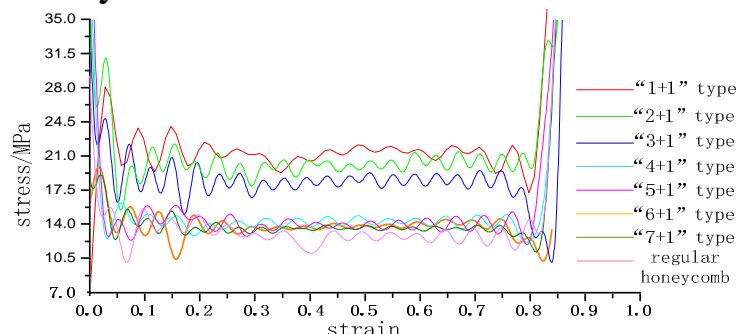

Fig. 10 Stress-strain curve of reinforced honeycomb in different superposition mode

The mean stress by simulation and theoretical analysis are comparatively analyzed as shown in Table 3.

Table 3 The simulation and theoretical analysis results

\begin{tabular}{|c|c|c|c|c|}
\hline superposition mode & $\begin{array}{c}\text { theoretical value } \\
(\mathrm{MPa})\end{array}$ & $\begin{array}{c}\text { Simulation value } \\
(\mathrm{MPa})\end{array}$ & $\begin{array}{c}\text { deviation of simulation and } \\
\text { theoretical value(\%) }\end{array}$ & $\begin{array}{c}\text { Simulation value } \\
\text { enhancement rate(\%) }\end{array}$ \\
\hline $1+1$ & 19.23 & 20.11 & 4.6 & 53.25 \\
\hline $2+1$ & 16.68 & 18.09 & 8.45 & 37.9 \\
\hline $3+1$ & 14.88 & 16.53 & 11.09 & 26 \\
\hline $4+1$ & 13.94 & 14.56 & 4.44 & 7.84 \\
\hline $5+1$ & 13.65 & 14.15 & 3.66 & 6.49 \\
\hline $6+1$ & 13.25 & 13.97 & 5.43 & 5.33 \\
\hline $7+1$ & 13.11 & 13.82 & 5.41 & - \\
\hline regular honeycomb & 11.93 & 13.12 & 9.97 & - \\
\hline
\end{tabular}

In view of Table 3, the strength of " $1+1$ " type is enhanced by $53.25 \%$ compared to the regular hexagonal honeycomb, and with the increase of the distance between the flat aluminum foil sheets, the strength of the honeycomb decreases gradually. The deviation of theoretical analysis and simulation result is between $3.66 \%$ and $11.09 \%$. Thereby the rationality of theoretical analysis is indicated.

\section{The experiment of axial quasi-static compression}

The material and method of experiment

Empirical tests are carried out on experimental samples of " $1+1$ ","4+1","7+1" type which are made of aluminum h5052, whose characteristic parameters are shown in Table 1. The size of samples in all are $100 \mathrm{~mm} * 100 \mathrm{~mm} * 100 \mathrm{~mm}$ in order to reduce effect induced by size variety which is shown in Fig. 11. All of them are pre-compressed to eliminate the peak force. The experiment of axial quasi-static compression is performed on the electronic universal testing machine in constant speed of $5 \mathrm{~mm} / \mathrm{min}$ to compress the honeycomb, as shown in Fig. 12. 


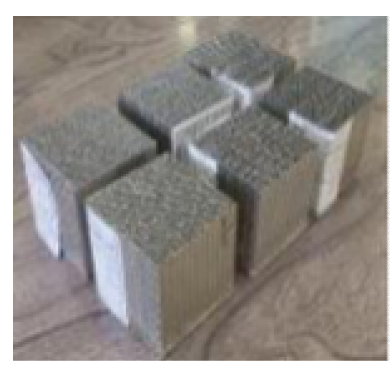

Fig. 11 Samples

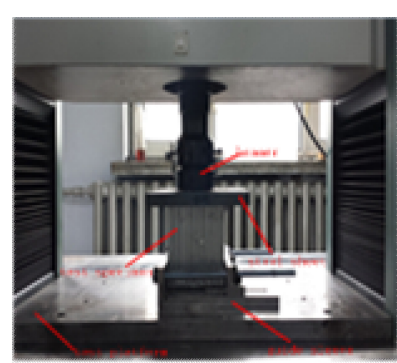

Fig. 12 Test platform

The experiment result analysis

As shown in Fig.13, the mean stress of "1+1" type reinforced honeycomb is highest in all experiment samples, but the fluctuation of the stress-stain curve is great. Corresponding to this, the curves of the " $4+1$ " and " $7+1$ "types are smooth and steady.

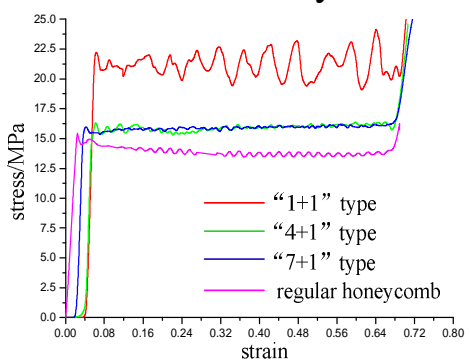

Figure 13 Stress-strain curve of different superposition mode honeycomb

Comparison of simulation and experiment

Two groups of tests are preformed to obtain the average value as the final result. The contrast of simulation analysis and experiment results is shown in Table 4:

Table 4 Comparison with the results of simulation and experiments

\begin{tabular}{|c|c|c|c|c|}
\hline superposition mode & $\begin{array}{c}\text { experiment value } \\
{[\mathrm{MPa}]}\end{array}$ & $\begin{array}{c}\text { simulation value } \\
{[\mathrm{MPa}]}\end{array}$ & $\begin{array}{c}\text { deviation of simulation and } \\
\text { experiment value[\%] }\end{array}$ & $\begin{array}{c}\text { experiment value } \\
\text { enhancement rate[\%] }\end{array}$ \\
\hline $1+1$ & 20.96 & 20.11 & 4.23 & 51.23 \\
\hline $4+1$ & 15.65 & 14.56 & 7.49 & 12.91 \\
\hline $7+1$ & 14.98 & 13.82 & 8.39 & 8.08 \\
\hline regular honeycomb & 13.86 & 13.12 & 5.32 & - \\
\hline
\end{tabular}

Table 4 further illustrates the relationship between reinforced honeycomb strength and superposition mode. The value of experiment is a little bigger than that of simulation, and the deviation is between $4.23 \%$ and $8.39 \%$. The experiment results confirm the validity of simulation model.

\section{Conclusion}

1). In this paper, varied reinforced honeycomb structures by changing the superposition mode of aluminum foil sheet was proposed, whose strength can be adjusted. The theoretical model of reinforced honeycomb was built to analyze the relationship of superposition mode and the mean stress based on the simplified basic folding element. The analysis results show that the mean stress of the honeycomb is related to the superposition mode, and it decreases with the increase of the aluminum flat foil spacer;

2). The simulation models of reinforced honeycomb for seven superposition modes were built under axial quasi-static compression to observe deformation mode and the process of compression. In addition, the simulation results are in good agreement with the theoretical analysis that verify the correctness of theoretical analysis.

3). Experiments of axial quasi-static compression of three superposition mode were implemented. The curves of stress-strain were obtained. By contrasting to the results of simulation and experiments, it is clear that two results coincide well. The validity of the simulated model and theoretical analysis are indicated. 


\section{References:}

[1].He Q, Ma D W. Parametric study and multi-objective crashworthiness optimisation of reinforced hexagonal honeycomb under dynamic loadings[J]. International Journal of Crashworthiness, 2015, 20(5):1-15.

[2].Yin Hanfeng, Wen Guilin. Crashworthiness Optimization Design of Honeycombs Based on the Simplified Basic Folding Element Method [J]. JOURNAL OF MECHANICAL ENGINNERING, 2011, 47(16):93-100.

[3].Wierzbicki T. Crushing analysis of metal honeycombs[J]. International Journal of Impact Engineering, 1983, 1(2):157-174.

[4].Li Meng. THE SIMULATION AND EXPERIMENT OF ALUMINUM HONEYCOMB AND FOAM ENERGY ABSORBERS FOR LEGGED LANDER[D]. Harbin Institute of Technology, 2009.

[5].Sun Deqiang, Sun Yujin, Zheng Bobo, et al. Simulation Analysis of the Out-of-plane Quasi-static Compression of Hexagonal Honeycomb Cores[J]. PACKING ENGINEERING, 2014(1):18-22.

[6].Bai Z, Guo H, Jiang B, et al. A study on the mean crushing strength of hexagonal multi-cell thin-walled structures[J]. Thin-Walled Structures, 2014, 80(9):38-45.

[7].Cai Mao, Gao Qun, Zong Zhijian. Energy Absorption Properties of Honeycomb Structure Aluminum under Axial Compression [J]. Journal of Material Science\&Engineering, 2015, 33(05):675-679.

[8].Liu Tianbao. Research on Assembly Process of High Strength Aluminum Honeycomb and Automotive Equipment[D]. Chongqing University of Technology, 2013.

[9].Chen W, Wierzbicki T. Relative merits of single-cell, multi-cell and foam-filled thin-walled structures in energy absorption[J]. Thin-Walled Structures, 2001, 39(4):287-306.

[10].Tang Shuang. The behavior of aluminum honeycomb under static and dynamic compression[D]. Central South University, 2014.

[11].Mahmoudabadi M Z, Sadighi M. A theoretical and experimental study on metal hexagonal honeycomb crushing under quasi-static and low velocity impact loading[J]. Materials Science \& Engineering A, 2011, 528(15):4958-4966.

[12].Zhang X W, Yu T X. Energy absorption of pressurized thin-walled circular tubes under axial crushing[J]. International Journal of Mechanical Sciences, 2009, 51(5):335-349.

[13].Luo Changjie, Zhou Anliang, Liu Rongqiang, et al. Average Compressive Stress Constitutive Equation of Honeycomb Metal under Out-of-plane Compression[J]. JOURNAL OF MECHANICAL ENGINNERING, 2010, 46(18):52-59.

[14].Yin Hanfeng. Crashworthiness optimization of energy absorbing structures for landing system[D]. Hunan University, 2011.

[15].Wierzbicki T, Abramowicz W. On the Crushing Mechanics of Thin-Walled Structures[J]. Journal of Applied Mechanics, 1983, 50(4):727-734.

[16].Wu E, Jiang W S. Axial crush of metallic honeycombs[J]. International Journal of Impact Engineering, 1997, 19(5-6):439-456. 